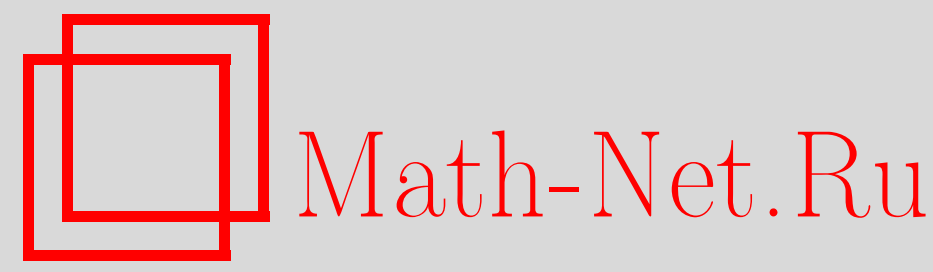

К. В. Емельянов, А. В. Цыгвинцев, Показатели Ковалевской систем с экспоненциальным взаимодействием, Матем. сб., 2000, том 191, номер 10, 39-50

DOI: https://doi.org/10.4213/sm514

Использование Общероссийского математического портала Math-Net.Ru подразумевает, что вы прочитали и согласны с пользовательским соглашением

http://www . mathnet.ru/rus/agreement

Параметры загрузки:

IP : 54.147 .182 .235

26 апреля 2023 г., $16: 34: 29$ 


\title{
Показатели Ковалевской систем с экспоненциальным взаимодействием
}

\begin{abstract}
Вычислены показатели Ковалевской обобщений цепочек Тоды - систем с экспоненциалным взаимодействием. Показано, что существующие случаи алгебраической интегрируемости не переносятся непосредственно на системы в пространстве с псевдоевклидовой метрикой, так как полнопараметрические разложения общего решения содержат комплексные степени независимой переменой.

Библиография: 15 названий.
\end{abstract}

\section{§1. Понятие систем с экспоненциальным взаимодействием}

Системой с экспоненииальным взаимодействием будем назьвать гамильтонову систему с гамильтонианом следующего вида:

$$
H=\frac{1}{2}(p, A p)+\sum_{i=1}^{N} \nu_{i} \exp \left(a_{i}, q\right)
$$

Здесь $p, q, a_{i} \in \mathbb{R}^{n},(\cdot, \cdot)$ - скалярное произведение в $\mathbb{R}^{n}, A: \mathbb{R}^{n} \rightarrow \mathbb{R}^{n}$ - симметрический оператор. Набор векторов $\Delta=\left\{a_{1}, \ldots, a_{N}\right\}$ назовем спектром системы. Мы следуем терминологии и обозначениям, принятым в [1].

Пусть $\langle\zeta, \xi\rangle \equiv(\zeta, A \xi)$. Введем в рассмотрение $(N \times N)$-матрицу $M$ с компонентами $M_{i k}=\left\langle a_{i}, a_{k}\right\rangle$. Уравнения движения

$$
\dot{q}=\frac{\partial H}{\partial p}, \quad \dot{p}=-\frac{\partial H}{\partial q}
$$

с помошью замены

$$
x_{i}=-\nu_{i} \exp \left(a_{i}, q\right), \quad y_{i}=\left\langle a_{i}, p\right\rangle
$$

преобразуем в систему $2 N$ дифференциальных уравнений с полиномиальными правьпи частями:

$$
\dot{x}_{k}=x_{k} y_{k}, \quad \dot{y}_{k}=\sum_{i=1}^{N} M_{k i} x_{i}, \quad 1 \leqslant k \leqslant N .
$$

Уравнения (3) допускают тривиальные первые интегралы

$$
F=\sum_{i=1}^{N} \alpha_{i} y_{i}, \quad \Phi=\sum_{i=1}^{N} x_{i}^{\alpha_{i}}
$$

(C) К. В. Емельянов, А. В. Цыгвинцев 
если сушествуют такие постоянные $\alpha_{i}$, что $\sum_{i=1}^{N} \alpha_{i} a_{i}=0$. При $N>n$ такие интегралы всегда сушествуют. Для движений, соответствующих исходной гамильтоновой системе, $F=0, \Phi=1$; при этом система (3) обладает интегралом энергии.

Гамильтоновы системы вида (1) впервые возникли в работе Тоды [2] при изучении межатомных взаимодействий. Позднее появились дальнейшие приложения этих уравнений, в частности, в космологии [3].

Механической моделью, описываемой системой (1), является цепочка взаимодействующих друг с другом экспоненциальным образом частиц единичной массы.

В работе [4] была установлена тесная связь между корневыми системами векторов алгебр Ли и некоторыми вполне интегрируемыми цепочками Тоды (1). Выяснилось, что возникающие при этом уравнения допускают естественное построение пары Лакса со спектральным параметром и, следовательно, могут быть проинтегрированы методами обратной задачи теории рассеяния [5].

Однако были найдены лишь некоторые случаи интегрируемости и вопрос их полной классификации оставался открытым. В работе [6] к анализу системы (2) был применен метод Ковалевской, впервые использованньй в динамике твердого тела [7]. Были найдены случаи, когда уравнения допускают максимально возможное число полнопараметрических мероморфных решений, названное В. В. Козловьгм числом Ковалевской.

В работе [8] был исследован вопрос интегрируемости системы (2) по Биркгофу. Рассматривались случаи, при которых эти уравнения обладают $n$ полиномиальньми по импульсам первыми интегралами с коэффициентами вида

$$
\sum_{k} f_{k} \exp \left(c_{k}, q\right)
$$

где $f_{k} \in \mathbb{R}, c_{k} \in \mathbb{R}^{n}$.

Полученные при этом ограничения на векторы спектра $\Delta$ выделяют уже найденные в работах [9], [6] случаи интегрируемости, а также предсказывают ряд новых, неизвестных ранее.

В настоящей работе мы находим все наборы показателей Ковалевской, соответствующие всевозможным решениям типа "квазиоднородного луча". Это открывает возможность исследования комплексно-аналитических свойств общего решения, в частности, получения необходимых условий его однозначности и мероморфности. Зная наборы показателей Ковалевской, можно делать предположения о виде первых интегралов, что может облегчить поиск интегрируемых систем. Возможно также рассмотреть вопрос обобщения известных результатов об интегрируемости на псевдоевклидов случай. К сожалению, ответ отрицательный. Существующие классы интегрируемых цепочек Тоды и их обобщений нельзя непосредственно перенести на псевдоевклидов случай.

\section{§2. Квазиоднородные системы ОДУ. Показатели Ковалевской}

Дадим определение квазиоднородной системы ОДУ.

ОПРЕДЕЛЕНИЕ 1. Систему уравнений

$$
\dot{x}_{i}=f_{i}\left(x_{1}, \ldots, x_{N}\right), \quad 1 \leqslant i \leqslant N,
$$


назовем квазиоднородной, если она инвариантна относительно преобразований

$$
x_{i} \rightarrow \alpha^{g_{i}} x_{i}, \quad t \rightarrow \alpha^{-1} t
$$

при произвольном $\alpha \neq 0$.

Постоянные $g_{i} \in \mathbb{Q}$ назовем весами квазиоднородности переменных $x_{i}$. Очевидно, что для квазиоднородности необходимо и достаточно, чтобы правые части уравнений удовлетворяли условиям

$$
f_{i}\left(\alpha^{g_{1}} x_{1}, \ldots, \alpha^{g_{N}} x_{N}\right)=\alpha^{g_{i}+1} f_{i}\left(x_{1}, \ldots, x_{N}\right) .
$$

Рассмотрим систему алгебраических уравнений

$$
-g_{i} c_{i}=f_{i}\left(c_{1}, \ldots, c_{N}\right), \quad 1 \leqslant i \leqslant N
$$

Если эти уравнения имеют ненулевые решения (возможно комплексные), то система (5) имеет однопараметрическое семейство мероморфных решений

$$
x_{i}=\frac{c_{i}}{\left(t-t_{0}\right)^{g_{i}}}, \quad t_{0}=\text { const } .
$$

Следуя работе [10], определим матрицу Ковалевской и показатели Ковалевской (KE).

ОПРЕДЕЛЕНИЕ 2. Матрица $K$ с компонентами

$$
K_{i j}=\frac{\partial f_{i}}{\partial x_{j}}(c)+g_{i} \delta_{i j}
$$

называется матрицей Ковалевской системы уравнений (5), вычисленной на векторе $c=\left(c_{1}, \ldots, c_{N}\right)^{T}$, соответствуюшем частным решениям (7). В формуле (8) $\delta_{i j}$ - дельта-символ Кронекера.

ОПРЕДЕЛЕНИЕ 3. Собственные числа матрицы Ковалевской называются $n$ оказателями Ковалевской.

Напомним, что функция $F\left(x_{1}, \ldots, x_{N}\right)$ называется квазиоднородной степени $m$, если для произвольного $\alpha$ справедливо:

$$
F\left(\alpha^{g_{1}} x_{1}, \ldots, \alpha^{g_{n}} x_{N}\right)=\alpha^{m} F\left(x_{1}, \ldots, x_{N}\right) .
$$

Векторное поле $v$ с компонентами $v_{i}$ назьвается полем симметрий системы (5), если линейные дифференциальные операторы

$$
\sum_{i=1}^{N} v_{i} \frac{\partial}{\partial x_{i}}, \quad \sum_{i=1}^{N} f_{i} \frac{\partial}{\partial x_{i}}
$$

коммутируют. При этом поле симметрий квазиоднородное степени $m$, если функции $v_{i} / x_{i}$ квазиоднородны степени $m$ каждая.

Матрица Ковалевской и показатели Ковалевской были введены в работе [10]. Их связь с тензорными инвариантами системы обсуждалась в [11]. Приведем две теоремы о квазиоднородных интегралах и полях симметрий уравнений (5). 
ТЕОРема 1 [10]. Если система уравнений (5) имеет $k$ первых интегралов степени однородности $m$, градиенты которых линейно независимы и не обращаются в нуль или бесконечность на решении системы (6), то $m$ - показатель Ковалевской кратности $k$ в наборе показателей, вычисленном для данного решения.

ТеОРема 2 [11]. Если система уравнений (5) имеет $k$ однородных полей симметрий степени однородности $m$, линейно независимых и не обращающихся в нуль или бесконечность на решении системы (6), то затель Ковалевской кратности $k$ в наборе показателей, вычисленном для данного решения.

СлЕДСТВИЕ 1. В каждом наборе показателей содержится -1. Если система гамильтонова, то в каждом наборе содержится степень однородности гамильтониана.

СлеДСТВИЕ 2. Для гамильтоновых систем показатели Ковалевской разбиваются на пары, сумма которых равна степени однородности гамильтониана минус 1 .

Показатели Ковалевской - степени независимой переменной $t$ в формальном полнопараметрическом разложении общего решения системы уравнений (5). K примеру, если $\rho$ - показатель Ковалевской, то уравнения имеют частное решение

$$
x_{i}=\frac{1}{t^{g_{i}}}\left(c_{i}+c_{i 1} t^{\rho}+c_{i 2} t^{2 \rho}+\cdots\right), \quad i=1, \ldots, n, \quad c_{k l} \in \mathbb{C}
$$

Подробное изложение связанных с этим вопросов можно найти в [12].

Если общее решение уравнений (5) однозначно на комплексной плоскости времени, то все показатели должны быть целыми числами. Наличие, к примеру, комплексных или иррациональных чисел в наборах показателей Ковалевской указывает на сложное строение римановой поверхности решения. Это замечание было использовано еще С. В. Ковалевской в ее работе [7], в которой она искала условия, при которых система дифференциальных уравнений Эйлера-Пуассона имеет общее решение, выраженное в мероморфных функциях времени. В работе [13] М. Адлер и П. ван Мербеке положили эту идею в основание определения алгебраической интегрируемости. Согласно М. Адлеру и П. ван Мербеке гамильтонова система

$$
\dot{z}=\{z, H\}, \quad z \in \mathbb{R}^{N}
$$

с полиномиальньм гамильтонианом $H$ и скобкой Пуассона $\{\cdot, \cdot\}$, вообще говоря, вырожденной, алгебраически интегрируема, если выполнены следующие условия.

1. Ограничение скобки на совместный уровень $k$ функций Казимира, коммутируюших со всеми $z_{i}$, невырождено.

2. Кроме функций Казимира $\Phi_{1}, \ldots, \Phi_{k}$ имеется $(N-k) / 2$ полиномиальных интегралов $\Phi_{k+1}, \ldots, \Phi_{k+n}=H$, множество их совместного уровня почти всегда компактно. 
3. Для почти всех комплексных значений первых интегралов можно найти многообразие $T_{\mathbb{C}}^{2 n} \backslash S$, где $T_{\mathbb{C}}^{2 n}=\mathbb{C}^{2 n} / \Gamma, \Gamma$ - решетка в $\mathbb{C}^{2 n}, S$ - объединение нескольких подмногообразий коразмерности один. Существуют $N$ абелевых функций, параметризующих комплексные инвариантные многообразия системы $\Lambda_{c}=\left\{z \in \mathbb{C}^{N}: \Phi_{i}=c_{i}, 1 \leqslant i \leqslant n+k\right\}$. На $T_{\mathbb{C}}^{2 n}$ можно выбрать естественные координаты $\zeta_{1}, \ldots, \zeta_{n}$, в которых фазовый поток на $\Lambda_{c}$ задается как $\dot{\zeta}=$ const. Отображение $T_{\mathbb{C}}^{2 n} \backslash S \rightarrow \Lambda_{c}$ взаимно однозначно.

В нашем случае для уравнений (3) в переменных $x, y$ скобки Пуассона следуюшие:

$$
\left\{y_{i}, x_{j}\right\}=\left\langle a_{i}, a_{j}\right\rangle x_{j}, \quad\left\{x_{i}, x_{j}\right\}=0, \quad\left\{y_{i}, y_{j}\right\}=0
$$

Интегралы (4) являются функциями Казимира. Примером алгебраически интегрируемых систем с экспоненциальным взаимодействием служат замкнутые цепочки Тоды:

$$
H=\frac{1}{2} \sum_{i=1}^{n} p_{i}^{2}+\sum_{i=1}^{n} \exp \left(q_{i}-q_{i+1}\right), \quad q_{n+1}=q_{1} .
$$

\section{§3. Показатели Ковалевской систем с экспоненциальным взаимодействием}

Показателями Ковалевской систем с әкспоненииальным взаимодействиeм назовем показатели Ковалевской соответствующих уравнений (3). Рассматриваемая система квазиоднородна с весами 2 и 1 по переменным $x$ и $y$ соответственно. Для нахождения частных решений вида (7) решим систему алгебраических уравнений

$$
-2 c_{k}=c_{k} d_{k}, \quad-d_{k}=\sum_{i=i}^{N} M_{i k} c_{i}, \quad 1 \leqslant k \leqslant N .
$$

Полагая все $c_{k}$, кроме одного, скажем $c_{i}$, равными нулю, находим решения:

$$
c_{k}=0, \quad c_{i}=\frac{2}{M_{i i}}, \quad d_{k}=-\frac{M_{k i}}{M_{i i}}, \quad d_{i}=0 .
$$

Аналогично, оставляя среди компонент вектора $c=\left(c_{1}, \ldots, c_{N}\right)^{T} m$ ненулевых компонент, получаем всевозможные решения вида

$$
\begin{gathered}
c_{j}=0, \quad\left\|\begin{array}{c}
c_{i_{1}} \\
\vdots \\
c_{i_{m}}
\end{array}\right\|=\left(M^{(m)}\right)^{-1}\left\|\begin{array}{c}
2 \\
\vdots \\
2
\end{array}\right\|, \\
d_{i_{1}=\cdots=d_{i_{m}}=}=-2, \quad d_{j}=\sum_{k \in\left\{i_{1}, \ldots, i_{m}\right\}} M_{j k} c_{k}, \\
j \notin\left\{i_{1}, \ldots, i_{m}\right\} .
\end{gathered}
$$

Здесь $M^{(m)}$ - соответствуюшая диагональная подматрица матрицы $M$ с элементами $M_{i j}, i, j \in\left\{i_{1}, \ldots, i_{m}\right\}$. Далее обозначим $c^{(m)}=\left(c_{i_{1}}, \ldots, c_{i_{m}}\right)^{T}$, 
$C^{(m)}=\operatorname{diag}\left(c_{i_{1}}, \ldots, c_{i_{m}}\right)$. Отметим также, что $M^{(m)}$ - матрица Грама системы векторов $\left\{a_{i_{1}}, \ldots, a_{i_{m}}\right\}$. При $m=1$ получаем решение (10).

Матрица Ковалевской для решения (11) имеет блочньй вид (для определенности пусть последние $m$ компонент $c=\left(c_{1}, \ldots, c_{N}\right)^{T}$ ненулевые):

$$
\begin{aligned}
K & =\left\|\begin{array}{cc}
U & C \\
M & E
\end{array}\right\| \\
U & =\operatorname{diag}\left(2+d_{1}, \ldots, 2+d_{N-m}, 0, \ldots, 0\right), \\
C & =\operatorname{diag}\left(0, \ldots, 0, c_{N-m+1}, \ldots, c_{N}\right), \\
E & =\operatorname{diag}(1, \ldots, 1) .
\end{aligned}
$$

Легко заметить, что имеются $N-m$ показателей Ковалевской, равных 1 , и $N-m$ показателей $2+d_{j}$. Последние назовем тривиальныцми и примем для них обозначение $\rho_{j}=2+d_{j}$. Оставшиеся $2 m$ нетривиальных показателей обозначим $\lambda$.

Лемма 1. Нетривиальные показатели разбиваются на пары $\lambda^{+}, \lambda^{-}$, являющиеся корнями уравнений вида $\lambda^{2}-\lambda-\beta=0$, одно из них $\lambda^{2}-\lambda-2=0, \beta$ - собственное значение задачи

$$
M^{(m)} \phi=\beta\left(C^{(m)}\right)^{-1} \phi
$$

ДокАЗАТЕЛЬство. Из (12) для нетривиальных показателей имеем

$$
M^{(m)} \phi+\varphi=\lambda \varphi, \quad C^{(m)} \phi=\lambda \phi
$$

где векторы $\phi=\left(\phi_{1}, \ldots, \phi_{m}\right)^{T}, \varphi=\left(\varphi_{1}, \ldots, \varphi_{m}\right)^{T}$ составлены из компонент собственного вектора матрицы Ковалевской $\left(0, \ldots, 0, \phi_{1}, \ldots, \phi_{m}, \ldots, \varphi_{1}, \ldots, \varphi_{m}\right)^{T}$, отвечаюшего собственному значению $\lambda$. Поэтому для нетривиальных показателей имеем следуюшую задачу на собственные значения:

$$
C^{(m)} M^{(m)} \phi=\beta \phi, \quad \beta=\lambda(\lambda-1) .
$$

Одно из решений $\phi=c^{(m)}, \beta=2$. Ему отвечают показатели $\lambda^{+}=2$ и $\lambda^{-}=-1$.

Отметим, что в наборах показателей, соответствующих решениям вида (10), тривиальными являются все, кроме $\lambda^{+}=2, \lambda^{-}=-1$. Этот набор был получен ранее в работе [1]. Там же поставлен вопрос, является ли равенство какого-либо показателя $\rho$ целому положительному числу достаточньм условием существования у системы (3) полиномиального интеграла соответствующей степени однородности. Мы видим, что ответ на этот вопрос отрицателен: необходимо наложить дополнительные условия на "высшие" наборы показателей Ковалевской, соответствуюшие решениям системы уравнений (11) с двумя и более ненулевыми $c_{k}$.

Итак, мы можем классифицировать наборы показателей в соответствии с числом $m$ ненулевых компонент вектора $c$. 
ТАБЛИЦА 1.

Наборы показателей Ковалевской систем с экспоненциальньм взаимодействием

\begin{tabular}{|c|c|}
\hline$m$ & Показатели Ковалевской \\
\hline 1 & $\left\{1, \ldots, 1, \rho_{1}, \ldots, \rho_{N-1},-1,2\right\}$ \\
2 & $\left\{1, \ldots, 1, \rho_{1}, \ldots, \rho_{N-2},-1,2, \lambda_{1}^{+}, \lambda_{1}^{-}\right\}$ \\
$\cdots$ & $\ldots$ \\
$n$ & $\left\{1, \ldots, 1, \rho_{1}, \ldots, \rho_{N-n},-1,2, \lambda_{1}^{+}, \lambda_{1}^{-}, \ldots, \lambda_{n-1}^{+}, \lambda_{n-1}^{-}\right\}$ \\
\hline
\end{tabular}

В общем случае количество различных наборов показателей в $i$-й строке табл. 1 равно количеству различных линейно независимых подсистем $i$ векторов из $\left\{a_{1}, \ldots, a_{N}\right\}$.

ПРИмеР 1. Рассмотрим гамильтониан

$$
H=\frac{1}{2} \sum_{i=1}^{n} p_{i}^{2}+\sum_{i=1}^{n-1} \exp \left(q_{i}-q_{i+1}\right)+\exp \left(-q_{1}-q_{2}\right)+\exp \left(q_{n}\right)+\exp \left(2 q_{n}\right), \quad n \geqslant 4 \text {. }
$$

В работе [1] выдвинуто предположение об интегрируемости соответствующих гамильтоновых уравнений. Вычисленные наборы показателей Ковалевской (табл. 2) содержат целые числа, при этом в каждом наборе не менее $n+1$ положительных, каждый из которых превосходит или равен 2. Принимая во внимание теоремы 1 и 2 , можно сказать, что проведенные авторами вычисления не опровергают предположения об интегрируемости системы с гамильтонианом (15). Предполагаемые степени однородности дополнительных первых интегралов $-4,6$ и 8.

ЛЕмма 2. Компоненты каждого вектора $c^{(m)}$ из (11) следующим образом связаны с показателями, вычисленными для векторов $c^{(m-1)}$ :

$$
c_{i}^{(m)}=\rho_{i}^{(m-1)} \frac{D^{(m-1)}}{D^{(m)}}
$$

где $\rho_{i}^{(m-1)}-$ тривиальный показатель одной из серий для $c^{(m-1)}, D^{(m-1)}=$ $\operatorname{det} M^{(m-1)}, D^{(m)}=\operatorname{det} M^{(m)}$.

Формула (16) справедлива, если показатели для $c^{(m-1)}$ существуют, т.е. в отсутствие вырождения $D^{(m)} \neq 0$.

Способом, приведенньм выше в данном параграфе, вычисляются все наборы показателей Ковалевской. Как уже отмечалось, наборы, соответствующие векторам $c=\left(c_{1}, \ldots, c_{N}\right)^{T}$ с одной ненулевой компонентой, были получены ранее в [1]. Равенство тривиальных показателей в таких наборах целым числам эквивалентно условию

$$
k\left\langle a_{i}, a_{i}\right\rangle+2\left\langle a_{i}, a_{j}\right\rangle=0, \quad i \neq j, \quad k \in \mathbb{Z},
$$

и необходимо для однозначности общего решения. Получив все оставшиеся наборы показателей и потребовав их целочисленности, мы существенно усиливаем эти необходимые условия. Для доказательства достаточности требуется дополнительный анализ, однако не исключено, что при полученных условиях решение дейст- 
ТАБЛИЦА 2.

Наборы показателей Ковалевской системы с гамильтонианом (15) при $n=4$

\begin{tabular}{|c|c|c|c|}
\hline Набор KE & Вектор $c$ & Набор KE & Вектор $c$ \\
\hline$\{1,1,1,1,1,1,2,2,2,2,2,2\}$ & $(0,0,0,0,0,0)$ & $\{-1,1,1,1,1,1,1,2,2,2,2,3\}$ & $(0,0,0,0,0,1)$ \\
\hline$\{-2,-1,1,1,1,1,1,2,2,2,2,4\}$ & $(0,0,0,0,1,0)$ & $\{-1,1,1,1,1,1,2,2,2,3,3,4\}$ & $(0,0,0,1,0,0)$ \\
\hline$\{-3,-1,1,1,1,1,1,2,2,2,4,5\}$ & $(0,0,0,3,0,4)$ & $\{-3,-2,-1,1,1,1,1,2,2,2,4,6\}$ & $(0,0,0,4,3,0)$ \\
\hline$\{-1,1,1,1,1,1,2,2,2,2,2,3\}$ & $(0,0,1,0,0,0)$ & $\{-1,-1,1,1,1,1,1,2,2,2,3,3\}$ & $(0,0,1,0,0,1)$ \\
\hline$\{-2,-1,-1,1,1,1,1,2,2,2,3,4\}$ & $(0,0,1,0,1,0)$ & $\{-1,-1,1,1,1,1,2,2,2,3,4,4\}$ & $(0,0,1,1,0,0)$ \\
\hline$\{-3,-1,-1,1,1,1,1,2,2,2,4,6\}$ & $(0,0,1,3,0,4)$ & $\{-3,-2,-1,-1,1,1,1,2,2,2,4,7\}$ & $(0,0,1,4,3,0)$ \\
\hline$\{-1,1,1,1,1,1,2,2,2,3,3,3\}$ & $(0,1,0,0,0,0)$ & $\{-1,-1,1,1,1,1,1,2,2,3,3,4\}$ & $(0,1,0,0,0,1)$ \\
\hline$\{-2,-1,-1,1,1,1,1,2,2,3,3,5\}$ & $(0,1,0,0,1,0)$ & $\{-2,-1,1,1,1,1,2,3,4,4,4,6\}$ & $(0,2,0,2,0,0)$ \\
\hline$\{-2,-1,1,1,1,1,2,2,2,3,4,4\}$ & $(0,2,2,0,0,0)$ & $\{-2,-1,-1,1,1,1,1,2,2,3,4,5\}$ & $(0,2,2,0,0,1)$ \\
\hline$\{-2,-2,-1,-1,1,1,1,2,2,3,4,6\}$ & $(0,2,2,0,1,0)$ & $\{-3,-2,-1,1,1,1,2,3,4,5,6,8\}$ & $(0,4,3,3,0,0)$ \\
\hline$\{-5,-3,-1,1,1,1,1,2,4,6,7,7\}$ & $(0,5,0,8,0,9)$ & $\{-5,-3,-2,-1,1,1,1,2,4,6,8,8\}$ & $(0,6,0,10,6,0)$ \\
\hline$\{-7,-5,-3,-1,1,1,1,2,4,6,8,14\}$ & $(0,12,7,15,0,16)$ & $\{-7,-5,-3,-2,-1,1,1,2,4,6,8,16\}$ & $(0,14,8,18,10,0)$ \\
\hline$\{-1,1,1,1,1,1,2,2,2,2,2,3\}$ & $(1,0,0,0,0,0)$ & $\{-1,-1,1,1,1,1,1,2,2,2,3,3\}$ & $(1,0,0,0,0,1)$ \\
\hline$\{-2,-1,-1,1,1,1,1,2,2,2,3,4\}$ & $(1,0,0,0,1,0)$ & $\{-1,-1,1,1,1,1,2,2,2,3,4,4\}$ & $(1,0,0,1,0,0)$ \\
\hline$\{-3,-1,-1,1,1,1,1,2,2,2,4,6\}$ & $(1,0,0,3,0,4)$ & $\{-3,-2,-1,-1,1,1,1,2,2,2,4,7\}$ & $(1,0,0,4,3,0)$ \\
\hline$\{-1,-1,1,1,1,1,2,2,2,2,2,4\}$ & $(1,0,1,0,0,0)$ & $\{-1,-1,-1,1,1,1,1,2,2,2,3,4\}$ & $(1,0,1,0,0,1)$ \\
\hline$\{-2,-1,-1,-1,1,1,1,2,2,2,4,4\}$ & $(1,0,1,0,1,0)$ & $\{-1,-1,-1,1,1,1,2,2,2,3,4,5\}$ & $(1,0,1,1,0,0)$ \\
\hline$\{-3,-1,-1,-1,1,1,1,2,2,2,4,7\}$ & $(1,0,1,3,0,4)$ & $\{-3,-2,-1,-1,-1,1,1,2,2,2,4,8\}$ & $(1,0,1,4,3,0)$ \\
\hline$\{-2,-1,1,1,1,1,2,2,2,3,4,4\}$ & $(2,2,0,0,0,0)$ & $\{-2,-1,-1,1,1,1,1,2,2,3,4,5\}$ & $(2,2,0,0,0,1)$ \\
\hline$\{-2,-2,-1,-1,1,1,1,2,2,3,4,6\}$ & $(2,2,0,0,1,0)$ & $\{-3,-2,-1,1,1,1,2,3,4,5,6,8\}$ & $(3,4,0,3,0,0)$ \\
\hline$\{-3,-2,-1,1,1,1,2,2,2,3,4,6\}$ & $(3,4,3,0,0,0)$ & $\{-3,-2,-1,-1,1,1,1,2,2,3,4,7\}$ & $(3,4,3,0,0,1)$ \\
\hline$\{-3,-2,-2,-1,-1,1,1,2,2,3,4,8\}$ & $(3,4,3,0,1,0)$ & $\{-5,-3,-3,-1,1,1,2,4,4,6,8,14\}$ & $(6,10,6,6,0,0)$ \\
\hline$\{-7,-5,-3,-1,1,1,1,2,4,6,8,14\}$ & $(7,12,0,15,0,16)$ & $\{-7,-5,-3,-2,-1,1,1,2,4,6,8,16\}$ & $(8,14,0,18,10,0)$ \\
\hline
\end{tabular}

вительно однозначно. Неясно также, обеспечивает ли равенство тривиальных показателей $\rho$ и нетривиальных $\lambda^{+}$неотрицательным цельм числам мероморфность общего решения и алгебраическую интегрируемость уравнений (3).

\section{§4. Алгебраическая неинтегрируемость псевдоевклидовых систем}

Пусть теперь матрица $A$ в гамильтониане (1) задает псевдоевклидову метрику

$$
\langle\zeta, \xi\rangle=-\sum_{i=1}^{\tau} \zeta_{i} \xi_{i}+\sum_{i=\tau}^{n} \zeta_{i} \xi_{i}, \quad 1 \leqslant \tau \leqslant n-1
$$

т.е. мы рассматриваем псевдоевклидово пространство $\mathbb{R}_{\tau}^{n}$. Следуя общепринятой терминологии, в зависимости от знака квадрата вектора мы называем векторы пространственноподобными $(\langle a, a\rangle>0)$, изотропными $(\langle a, a\rangle=0)$ и времениподобными.

Справедливо следующее утверждение.

ТЕОрема 3. Пусть в гамильтониане (1) спектр $\Delta=\left\{a_{1}, \ldots, a_{N}\right\}$ maков, что найдется подсистема $\Delta_{-} \subset \Delta$ времениподобных векторов, линейная оболочка которых содержст пространственноподобный вектор или, 
наоборот, некоторая линейная оболочка пространственноподобных векторов $\Delta_{+} \subset \Delta$ содержит времениподобный вектор. Тогда если для любых $a_{i}, a_{j} \in \Delta_{ \pm}$справедливо условие

$$
k\left\langle a_{i}, a_{i}\right\rangle+2\left\langle a_{i}, a_{j}\right\rangle=0, \quad i \neq j, \quad k=0,1,2 \ldots,
$$

то существует набор показателей Ковалевской, содержсащий комплексно-сопряженные числа с ненулевой мнимой частью.

ДокАЗАтЕльство. Допустим, что имеется несколько пространственноподобных векторов таких, что натянутое на них линейное пространство содержит времениподобные векторы.

Пусть $\Delta_{+}=\left\{a_{1}, \ldots, a_{m}\right\}, \Delta_{+} \subset \Delta,-$ минимальный набор, удовлетворяющий условиям теоремы. Рассмотрим матрицу Грама $\left\|M_{i j}^{(m)}\right\|=\left\|\left\langle a_{i}, a_{j}\right\rangle\right\|$ для набора $\Delta_{+}$. По условию теоремы такая матрица знаконеопределена. Учитывая лемму 1 , для доказательства необходимо показать, что все числа $c_{j}$, являющиеся решением линейных алгебраических уравнений

$$
M_{i j}^{(m)} c_{j}=2, \quad i, j=1, \ldots, m
$$

имеют один знак, т.е. матрица $C^{(m)}=\operatorname{diag}\left(c_{1}, \ldots, c_{m}\right)$ знакоопределена. Доказательство состоит в прямом вычислении компонент $c_{i}$. Применяя правило Крамера и разлагая соответствующие определители по $i$-й строке, придем к тому, что из условий (18) следует отрицательность всех $c_{i}$, что доказывает сушествование отрицательного собственного значения $\beta$ задачи (13). То, что существует $\beta<-\frac{1}{4}$, следует из известных теорем алгебры об инвариантах квадратичных форм. Для доказательства в случае $\Delta_{-}$достаточно рассмотреть гамильтониан $-H$.

В работе [8] показано, что несоблюдение условий (18) для евклидовой метрики $\langle\cdot, \cdot\rangle$ приводит к несуществованию у гамильтоновой системы (1) в случае $n=N$ достаточного количества первых интегралов, полиномиальных по импульсам с коэфффициентами вида:

$$
\sum_{k} f_{k} \exp \left(c_{k}, q\right), \quad f_{k} \in \mathbb{R}, \quad c_{k} \in \mathbb{R}^{n}
$$

В [4] рассматривались системы с экспоненциальньм взаимодействием в евклидовой метрике при $N=n+1$ в предположении, что в наборе векторов $\left\{a_{1}, \ldots, a_{N}\right\}$ любые $n$ линейно независимы. Показано, что для таких систем условия (18) являются критерием алгебраической интегрируемости. Обобшение этого результата можно найти в [1]. У тверждение теоремы 3 означает, в частности, что полученные для евклидовой метрики результаты не переносятся непосредственно на псевдоевклидов случай. Заметим, что если $\langle\cdot, \cdot\rangle$ - евклидова метрика, то справедливо обратное доказанной теореме: из условий (18) следует действительность показателей Ковалевской.

С использованием теоремы 3 легко доказывается следующее утверждение. 
ТЕОРема 4. Пусть в гамильтониане (1) $N=n,\langle\cdot, \cdot\rangle-$ псевдоевклидова метрика (17). Если множество векторов $\left\{a_{1}, \ldots, a_{n}\right\}$ образует базис и среди них содержится $\tau+1$ времениподобный или $n-\tau+1$ пространственноподобный, то соответствующие уравнения (3) не имеют полного набора полиномиальных интегралов, градиенты которых линейно независимы и не обращаются в нуль или бесконечность на решениях (10), т.е. удовлетворяют условиям теоремы 1 для наборов показателей в первой строке табл. 1.

ДокАЗАТЕЛьство. Из линейной независимости векторов $a_{1}, \ldots, a_{n}$ следует несуществование интегралов первой степени однородности (все они имеют вид $F$ из (4)). Степени однородности отличных от гамильтониана интегралов содержатся среди тривиальных показателей Ковалевской из первой строки табл. 1. Отсюда следует справедливость условий (18) и сушествование комплексно-сопряженных показателей, что несовместимо с алгебраической интегрируемостью и существованием полного набора полиномиальных интегралов.

Рассмотрим гамильтонову систему (1) в пространстве Минковского $\mathbb{R}_{1}^{n}$, положив $N=n$ :

$$
H=\frac{1}{2}\langle p, p\rangle+\sum_{i=1}^{n} \nu_{i} \exp \left(a_{i}, q\right) .
$$

Поставим вопрос о сушествовании у этой системы полного набора полиномиальных по $p, \nu_{k} \exp \left(a_{k}, q\right)$ интегралов, удовлетворяющих условиям теоремы 1 для первой серии показателей из табл. 1. Для таких интегралов $H=I_{1}, I_{2}, \ldots, I_{n}$ :

$$
\begin{aligned}
& d I_{k} \neq 0, \infty \text { на решениях }(10), \\
& d I_{1}, d I_{2}, \ldots, d I_{n} \text { линейно независимы на решениях }(10) .
\end{aligned}
$$

По теореме 1 степень однородности полиномов $I_{1}, I_{2}, \ldots, I_{n}$ содержится среди показателей Ковалевской, интегралов вида (3) не существует, поскольку мы предполагаем линейную независимость векторов спектра, следовательно, тривиальные показатели - положительные целые числа, превосходящие 1. Это эквивалентно условию (18).

Исключим из рассмотрения случаи, когда уравнения Гамильтона разбиваются на $l$ замкнутых гамильтоновых систем с функциями Гамильтона $H_{i}$, т.е. будем рассматривать неприводимые системы.

Спектр $\Delta$ системы (19) всегда можно представить в виде объединения $\Delta=\Delta_{+} \cup$ $\Delta_{-} \cup \Delta_{0}$, где $\Delta_{+}, \Delta_{-}, \Delta_{0}$ - множества пространственноподобных, времениподобных и изотропных векторов соответственно. Применяя доказанные теоремы, мы приходим к следующему утверждению.

ТЕОРема 5. Спектр неприводимой интегрируемой в смысле условий (20) системы (19), не содержащей изотропных векторов, совпадает с одним из многеств $\Delta_{+}, \Delta_{-}$.

Мы исключили из рассмотрения изотропные векторы спектра, поскольку наш анализ базируется на условии (18), являющемся условием положительности тривиальных показателей Ковалевской в наборах первой серии (первая строка табл. 1). В случае, если имеется изотропный вектор: $\langle a, a\rangle=0$, соответствующего 
набора показателей просто не сушествует, условие (18) неприменимо и необходимо рассматривать "высшие" наборы.

Отметим, что наличие комплексно-сопряженных показателей Ковалевской в некоторых конкретных псевдоевклидовых системах с экспоненциальным взаимодействием ранее отмечалось в литературе при исследовании космологических моделей [14].

\section{§5. Заключение}

Исследование показателей Ковалевской, предложенное Х. Иошидой в работе [10], явилось весьма эффективньпм методом исследования квазиоднородных систем. По сути, в этом случае мы имеем возможность легко провести так называемьй тест Ковалевской-Пенлеве на однозначность и мероморфность обшего решения. В последнее время появилось много работ, обобщающих и развивающих эту методику. Используя арифметические свойства показателей, В. В. Козлов и С. Д. Фурта в [12] доказали ряд теорем о неинтегрируемости целого класса "полуквазиоднородных" систем. Ранее сильный результат о неинтегрируемости гамильтоновых квазиоднородных систем был получен тем же Х. Иошидой [15]. Немаловажно также, что вычисленные показатели Ковалевской содержат в себе информацию о степенях квазиоднородности дополнительных первых интегралов и полей симметрий, что делает этот метод очень привлекательным не только для доказательства неинтегрируемости, но и при поиске интегрируемых случаев. Данная работа, как мы надеемся, иллюстрирует это: мы получили результаты о неинтегрируемости псевдоевклидовых систем и высказали предположение о степенях однородности дополнительных интегралов системы (15).

Авторы благодарят Д.В. Трещева, В. В. Козлова за полезные обсуждения.

\section{Список литературы}

1. Козлов В. В. Симметрии, топология и резонансы в гамилтоновой механике. Ижевск: Изд-во УдГУ, 1995.

2. Тода М. Теория нелинейных решеток. М.: Мир, 1984.

3. Богоявленский О. И. Методы качественной теории динамических систем в астрофизике и газовой динамике. М.: Наука, 1980.

4. Adler M., van Moerbeke P. Kowalewski's asymptotic method, Kac-Moody Lie algebras and regularization // Comm. Math. Phys. 1982. V. 83. P. 83-106.

5. Арнольд В.И., Козлов В. В., Нейштадт А. И. Математические аспекты классической и небесной механики // Итоги науки и техники. Совр. пробл. матем. Фундам. напр. Т. 3. М.: ВИНИТИ, 1985.

6. Козлов В. В., Трещев Д. В. Числа Ковалевской обобщенных цепочек Тоды // Матем. заметки. 1989. Т. 46. № 5. С. 17-28.

7. Ковалевскал С. В. Задача о вращении твердого тела около неподвижной точки // Ковалевская С. В. Научные работы. М.: Изд-во АН СССР, 1948. С. 153-220.

8. Козлов В. В., Трещев Д. В. Полиномиальные интегралы гамильтоновых систем с экспоненциальньм взаимодействием // Изв. АН СССР. Сер. матем. 1989. Т. 51. №3. C. $537-556$.

9. Adler M., van Moerbeke P. The Toda lattice, Dynkin diagrams, singularities and Abelian varietes // Invent. Math. 1991. V. 103. P. 223-278.

10. Yoshida $H$. Necessary condition for the existence of algebraic first integrals // Celestial Mech. 1983. V. 31. P. 363-399. 
11. Козлов В. В. Тензорные инварианты квазиоднородных систем дифференциальных уравнений и асимптотический метод Ковалевской-Ляпунова // Матем. заметки. 1992. Т. 51. № 2. C. $46-52$.

12. Козлов В.В., Фурта С.Д. Асимптотики решений сильно нелинейных систем дифференциальных уравнений. М.: Изд-во МГУ, 1996.

13. Adler M., van Moerbeke P. A systematic approach towards solving integrable systems // Perspectives in Mathematics. New York: Academic Press, 1987.

14. Pavlov A. E. The mixmaster cosmological model as a pseudo-Euclidean generalized Toda chain // Regular and Chaotic Dynamics. 1996. № 1. P. 111-118.

15. Yoshida $H$. A criterion for the non-existence of an additional analytic integral in Hamiltonian systems with $n$ degrees of freedom // Phys. Lett. A. 1989. V. 141. № 3-4. P. 108-112.

Удмуртский государственный университет;

Московский государственнњй университет им. М.В. Ломоносова

E-mail: ke@uni.udm.ru, tsygvin@nw.math.msu.su 